Supporting Information

\title{
Molecular Dynamics Simulation of \\ Emulsification/Demulsification with a Gas Switchable Surfactant
}

Xiangliang Liu, ${ }^{\dagger}$ Y ingjie Li, ${ }^{\dagger}$ Senlin Tian ${ }^{*}{ }^{\dagger}$ and Hui Yan, ${ }^{*}$,

${ }^{\dagger}$ Faculty of Environmental Science and Engineering, Kunming University of Science and Technology, Kunming 650500, China

\$School of Pharmacy, Liaocheng University, Liaocheng 252059, China

*E-mail: yanhuilcu@163.com (H.Y.); tiansenlin@outlook.com (S.T.).

\section{Table of Contents}

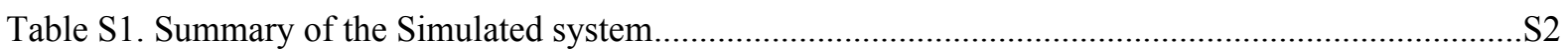

Figure S1. Configurations of the simulated systems with different surfactant-hexadecane proportion ......S3

Figure S2. Number density distribution profile for the other emulsion droplet from system I.....................S4

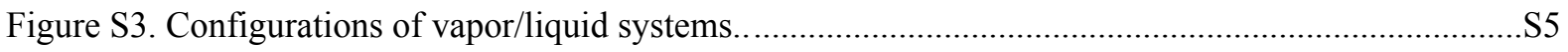

Figure S4. Conformational change of the oil droplet at the early period of the simulation..........................S6

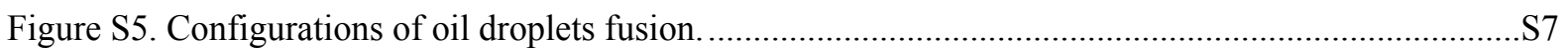

Figure S6. Distance variation and configurations of the emulsion droplets coalescence. ............................S8 
Table S1. Summary of the Simulated system

\begin{tabular}{ccccccc}
\hline \hline system label & oil & DDA & $\mathrm{DDA}^{+}$ & $\mathrm{HCO}^{-}$ & water & $\begin{array}{c}\text { simulation } \\
\text { time }\end{array}$ \\
\hline I or IV & 300 & 100 & & & 26299 & $10 \mathrm{~ns}$ \\
II or V & 300 & & 100 & 100 & 25944 & $100 \mathrm{~ns}$ \\
III & 300 & 100 & & & 25944 & $10 \mathrm{~ns}$ \\
\hline SMD & 364 & 126 & & & 54635 & \\
simulation & 364 & & 126 & 126 & 54252 & \\
\hline \hline
\end{tabular}




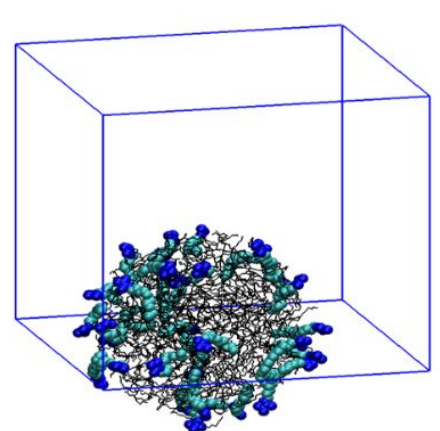

$1: 6$

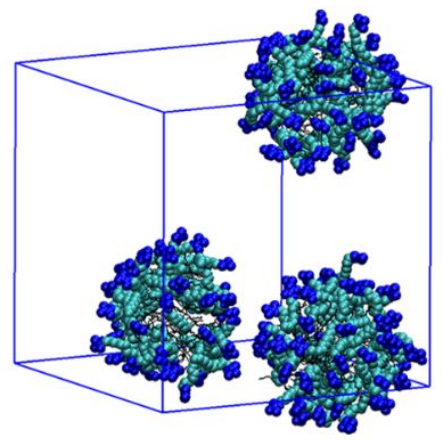

$1: 1$

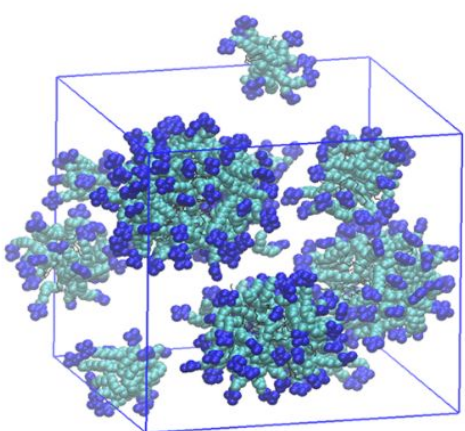

$3: 1$

Figure S1. Final configurations of the simulated systems with different surfactant-hexadecane proportion. The numbers of $\mathrm{DDA}^{+}$:hexadecane for these three systems are, in turn, 50:300, 150:150, and 300:100. 


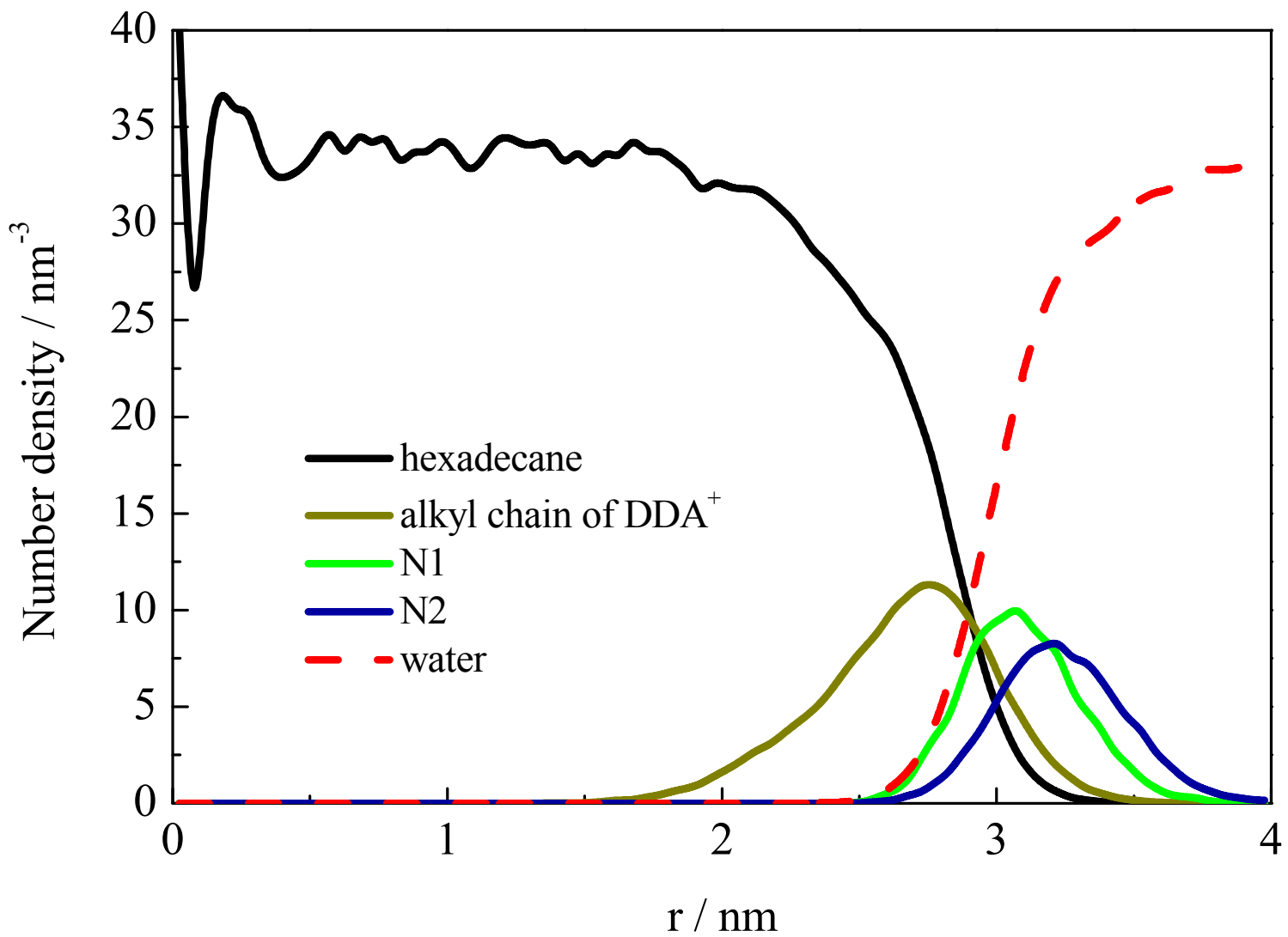

Figure S2. Number density distribution of components with respect to COM of the other larger droplet obtained from system I. 


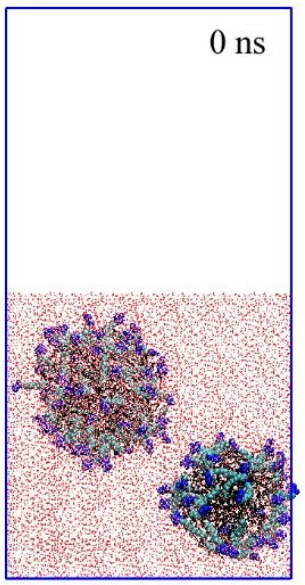

(a)

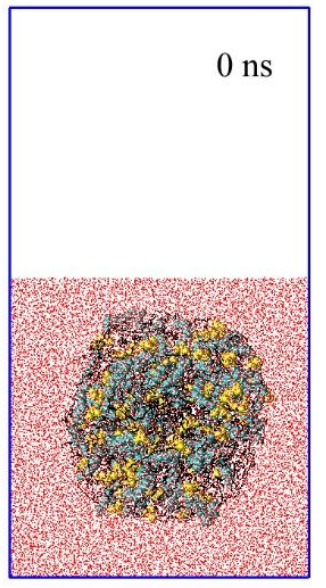

(c)

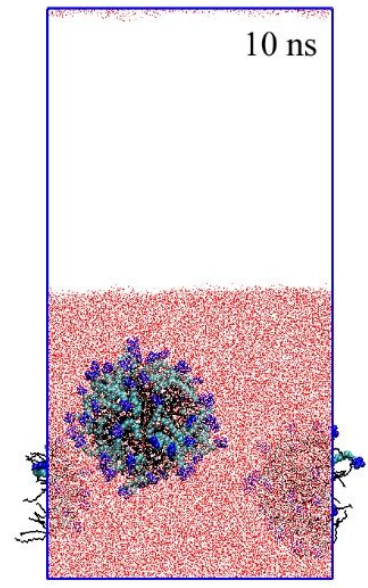

(b)

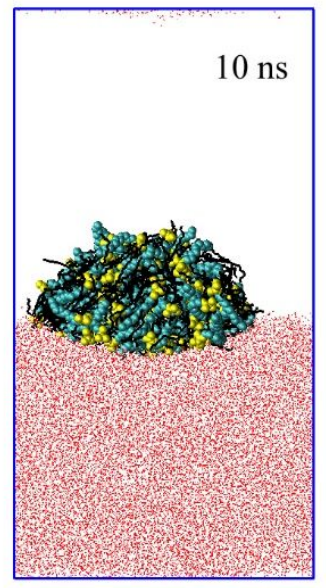

(d)

Figure S3. Configurations of vapor/liquid systems. The display mode for the molecules is identical to that in Figure 1. 

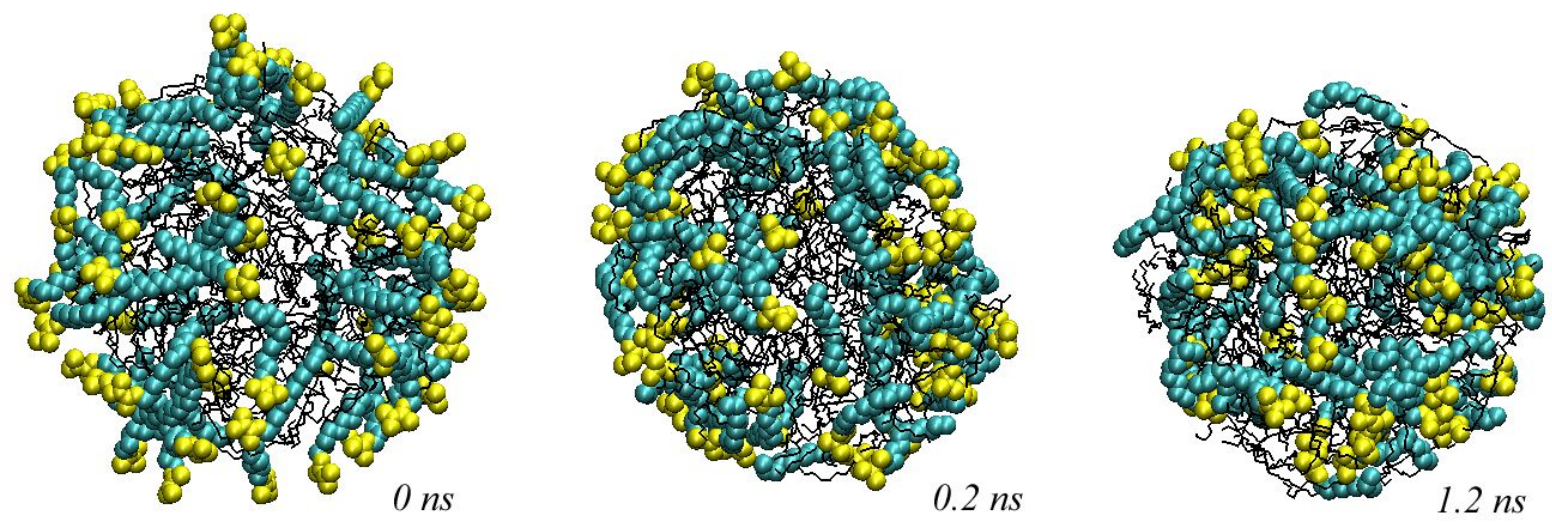

Figure S4. Conformational change of the oil droplet at the early period of the simulation when the $\mathrm{DDA}^{+}$surfactants were changed into DDA molecules. 


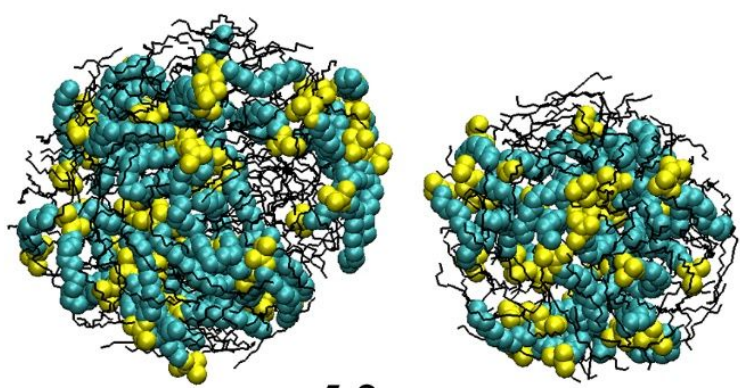

$5.2 \mathrm{~ns}$

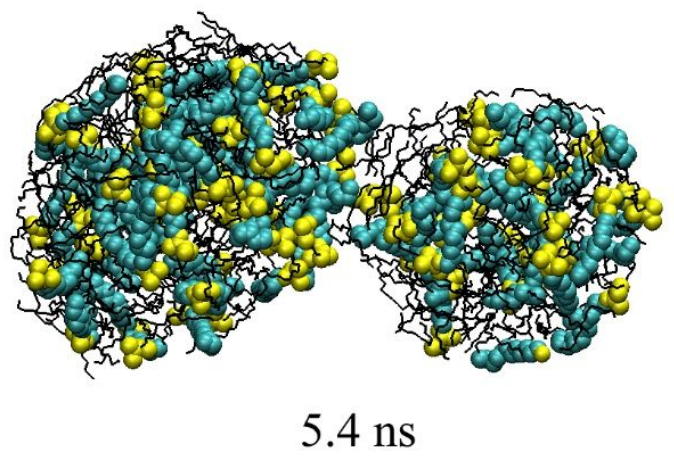

Figure S5. Configurations of oil droplets fusion. 

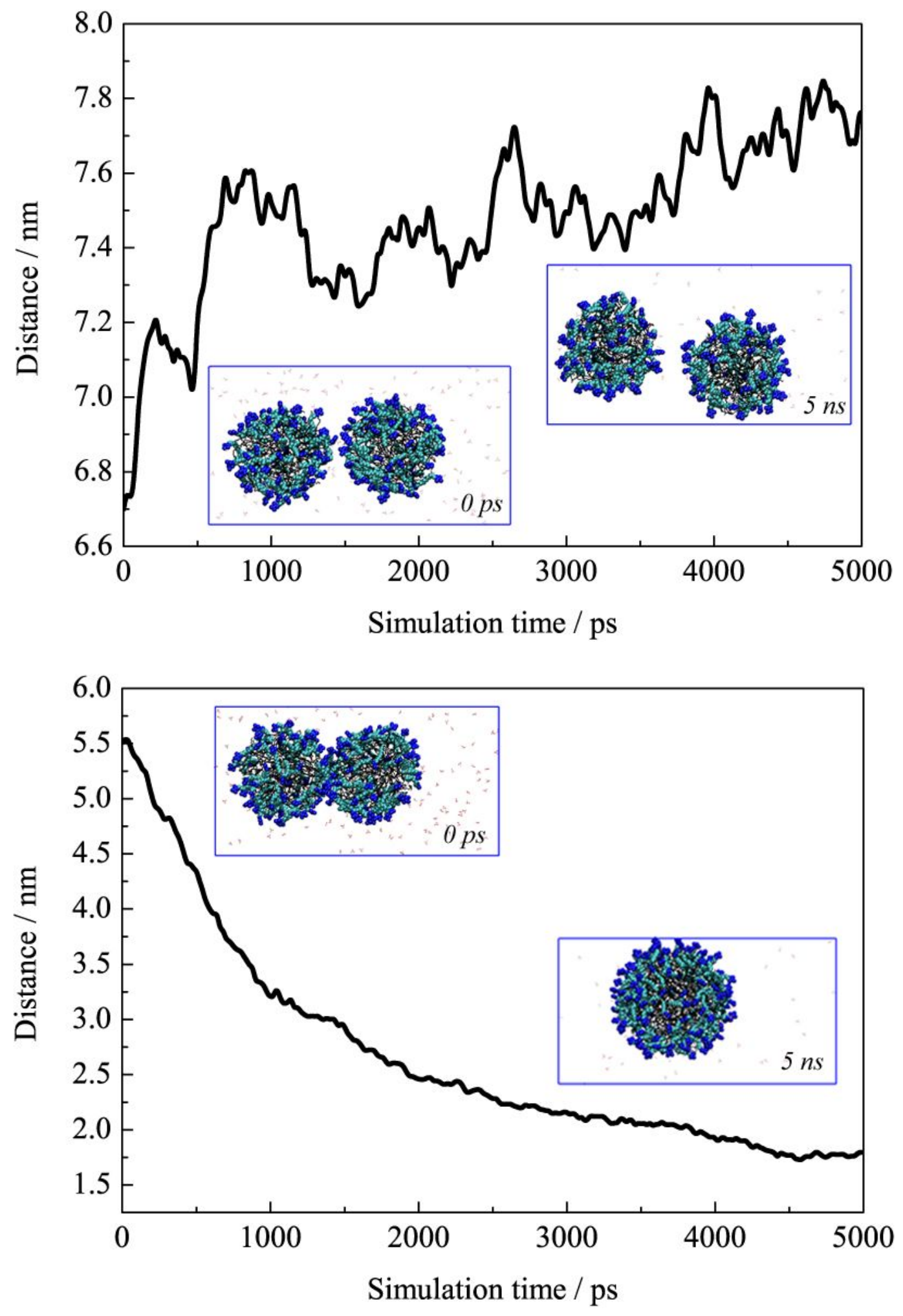

Figure S6. Distance variation and configurations of the emulsion droplets coalescence. 